\title{
Message from the Conference Chairman
}

On behalf of the ISEM 2017 Organizing Committee, we are proud to publish selected contributions presented at the 18th International Symposium on Applied Electromagnetics and Mechanics (ISEM 2017) that was held successfully from September 3rd to 6th, 2017 at the Majestic - Palais des Congrès, Chamonix Mont-Blanc, France. The first symposium of the series took place in Japan in 1988, and, since then, they have continued to grow in numbers, significance, and international renown and respect, wherever their location, including Korea, the United Kingdom, Germany, Italy, France, Austria, the USA, China and Canada. We are honored, therefore, to pursue what has become a well-established tradition of presenting leading research and ideas in the area of applied electromagnetics and mechanics.

For ISEM 2017 in Chamonix Mont-Blanc, 380 contributions were submitted to the editorial board, of which 351 were accepted for presentation. More than 310 participants, including 79 students, from 22 countries attended Chamonix Mont-Blanc for the symposium! For this special issue of the International Journal of Applied Electromagnetics and Mechanics, the editorial committee received 247 extended papers and, after a painstaking review by the referees, 177 have been selected for publication. These record numbers show the significant interest and great importance of the topics covered by ISEM.

Many people contributed to the success of ISEM 2017 in Chamonix Mont-Blanc. I would especially like to thank the Organizing Committee: Afef Kedous-Lebouc, Nora Dempsey, Riccardo Scorretti and Marc Lambert who served as co-chairs. Anne Pellissier, Géraldine Pignatelli, Corinne Matarasso and Damien Corral did a great job in organizing the invitation process, venue and accommodation, design and graphic chart, and the website (http://www.isem2017.org/). And of course, none of this would have been possible without the efficient just-in-time management provided by Catherine Auguet-Chadaj and the hosting of MINES ParisTech. The ISEM International Steering Committee, chaired by Toshiyuki Takagi, and the organizers of ISEM 2015 in Kobe (Japan) were also very helpful, providing us with valuable information and advice. The editorial board chaired by Christophe Geuzaine did an outstanding job in reviewing papers for the conference. Lastly, we are of course grateful to all of our colleagues who helped us as reviewers.

Finally, I would like to thank our principal sponsors: the Centre National de la Recherche Scientifique (France), the Universite Grenoble-Alpes and its engineering school Grenoble-INP (France), the Japan Society for the Promotion of Science, the Rhône-Alpes-Auvergne Region, the City of Chamonix MontBlanc, and the companies Altair and Schneider Electric.

Vincent Mazauric

Chairman of ISEM 2017 in Chamonix Mont-Blanc

Schneider Electric (Grenoble) and MINES ParisTech (Sophia-Antipolis) France 
ISEM 2017 Editorial Board

Program Chair: Christophe Geuzaine (Institut Montefiore, Liege, Belgium)

Track Chairs:

- Afef Lebouc (G2Elab, Grenoble, France)

- Nora Dempsey (Institut Néel, Grenoble, France)

- Riccardo Scorretti (Ampère, Lyon, France)

- Marc Lambert (GeePs, Gif-sur-Yvette, France)

- Dominique Lesselier (L2S, Gif-sur-Yvette, France)

$\begin{array}{ll}\text { Jérémie Aimé } & \text { Edouard Demaldent } \\ \text { Farid Allab } & \text { Ping-Ping Ding } \\ \text { Morgan Almanza } & \text { Oliver Dorn } \\ \text { Yacine Amara } & \text { Yves Du Terrail Couvat } \\ \text { Laure Arbenz } & \text { Frédéric Dubas } \\ \text { Aktham Asfour } & \text { Benjamin Ducharne } \\ \text { Yvan Avenas } & \text { Stéphane Dufour } \\ \text { Georges Barakat } & \text { Christophe Espanet } \\ \text { Michel Barrault } & \text { Richard Evans } \\ \text { Skandar Basrour } & \text { Aurelie Fasquelle } \\ \text { Anouar Belahcen } & \text { Marylin Fassenet } \\ \text { Cedric Bellis } & \text { Mouloud Feliachi } \\ \text { Mohamed Benbouzid } & \text { Julien Fontchastagner } \\ \text { Kevin Berger } & \text { Radu Fratila } \\ \text { Sandor Bilicz } & \text { Raffaele Fresa } \\ \text { Marc Bonnet } & \text { Nicolas Galopin } \\ \text { Frederic Bouillault } & \text { Lauric Garbuio } \\ \text { Pascal Brochet } & \text { Olivier Geoffroy } \\ \text { François Buret } & \text { Frederic Gillon } \\ \text { Caifang Cai } & \text { Leticia Gimeno } \\ \text { Olivier Chadebec } & \text { Gilles Guerassimoff } \\ \text { Tomasz Chady } & \text { Maya Hage-Hassan } \\ \text { Jean-Frédéric Charpentier } & \text { Rachelle Hanna } \\ \text { Hervé Chazal } & \text { Laurent Hirsinger } \\ \text { Zhenmao Chen } & \text { Sami Hlioui } \\ \text { Fengjun Chen } & \text { Emmanuel Hoang } \\ \text { Ahmed Cheriet } & \text { Xiao Hua } \\ \text { Laurence Cheze } & \text { Olivier Hubert } \\ \text { Christian Chillet } & \text { Hajime Igarashi } \\ \text { Vincenzo Cirimele } & \text { Olivier Isnard } \\ \text { Romain Corcolle } & \text { Amit Jha } \\ \text { Olivier De La Barrière } & \text { Hongli Ji } \\ \text { Thierry De Larochelambert } & \text { Laurent Jossic } \\ \text { Yves Delannoy } & \text { Pierre-Yves Joubert } \\ \text { Francesco Dell'Isola } & \text { Andrej Kitanovski } \\ & \end{array}$

Jérémie Aimé

Morgan Almanza

Yacine Amara

Laure Arbenz

Aktham Asfour

Anouar Belahcen

Cedric Bellis

Mohamed Benbouzid

Frederic Bouillault

Pascal Brochet

Tomasz Chady

Jean-Frédéric Charpentier

Ahmed Cheriet

Laurence Cheze

Olivier De La Barrière

Amit Jha

Andrej Kitanovski
Fumio Kojima

Erin Kuci

Nicolas Labbe

Vincent Lanfranchi

Vladimir Lazarov

Yann Le Bihan

Yvan Lefevre

Ziwei Li

Changyou Li

Jean-Francois Llibre

Elena Lomonova

Olivier Maloberti

Patrick Mas

Daniel Matt

Gérard Meunier

Oualid Messal

Jonas Millinger

Roberto Miorelli

Rachid Missoum

Laurent Morel

Anthony Moses

Sergio Muhlen

Takahisa Ohji

Florence Ossart

Jozsef Pavo

Sylvain Perez

Ronan Perrussel

Mickael Petit

Laureline Porcar

Denis Premel

Carlo Ragusa

Brahim Ramdane

Peter Rasmussen

Almpion Ratsakou

Marie-Ange Raulet
Christophe Reboud

Maurizio Repetto

Abderrazek Rezzoug

Daniel Roger

Laure-Line Rouve

Andrew Rowe

Vincent Russier

Ruth Sabariego

Sheppard Salon

Tatsuo Sawada

Hans Schellekens

Philippe Schuster

Gael Sebald

Georgi Shilyashki

Fabien Sixdenier

Anastasios Skarlatos

Juliette Soulard

Toshiyuki Takagi

Antonello Tamburrino

Julio Teixeira

Theodoros Theodoulidis

André Thiaville

Mounain Tounzi

Pranshu Upadhayay

Jean-Claude Vannier

Gérard Vinsard

Eric Vourch

Guillaume Wasselynck

Shejuan Xie

Shiyou Yang

Noritaka Yusa

Yu Zhong

Salah-Eddine Zouzou 